\title{
Computation of Composition Functions and Invariant Vector Fields in Terms of Structure Constants of Associated Lie Algebras
}

\author{
Alexey A. MAGAZEV, Vitaly V. MIKHEYEV and Igor V. SHIROKOV \\ Omsk State Technical University, 11 Mira Ave., Omsk, 644050, Russia \\ E-mail: magazev@gmail.com,vvm125@mail.ru,iv_shirokov@mail.ru
}

Received December 05, 2013, in final form July 25, 2015; Published online August 06, 2015

http://dx.doi.org/10.3842/SIGMA.2015.066

\begin{abstract}
Methods of construction of the composition function, left- and right-invariant vector fields and differential 1-forms of a Lie group from the structure constants of the associated Lie algebra are proposed. It is shown that in the second canonical coordinates these problems are reduced to the matrix inversions and matrix exponentiations, and the composition function can be represented in quadratures. Moreover, it is proven that the transition function from the first canonical coordinates to the second canonical coordinates can be found by quadratures.
\end{abstract}

Key words: Lie group; Lie algebra; left- and right-invariant vector fields; composition function; canonical coordinates

2010 Mathematics Subject Classification: 22E05; 22E60; 22E70

\section{Introduction}

Researchers in the field of theoretical and mathematical physics who use methods of Lie theory face the problem on realizations of a finite-dimensional Lie algebra by means of vector fields on a certain domain of a finite-dimensional real space. This problem is vitally important for group classification of partial differential equations [3, 13], for the classification of pseudoRiemannian metrics on manifolds with groups of motions [20], as well as for the construction of relativistic wave equations in external fields with a given symmetry group [14]. The more general and interesting problem on realizations of Lie algebras by nonhomogeneous first-order differential operators should also be mentioned in this context. It naturally emerges in the theory of projective representations of Lie groups [2, 17]. This problem is of great importance in applications, for instance in quantum theory of scattering [1] and in integration of differential and integro-differential equations [9, 23].

The problem on realizations of a Lie algebra by vector fields has long history and goes back to works of S. Lie but modern mathematicians still demonstrate their interest to this field and their approaches are directly depend on applications. As a result, now there are a sufficiently large number of works in this field. We point out some of the most important results.

Undoubtedly, S. Lie stated the principal ideas in this field, and the first important results also belong to him. For example, he listed all possible realizations of finite-dimensional Lie algebras on the real and complex lines. Later he presented the similar result for the complex plane [15]. The results of S. Lie were completed by the classification of vector fields on the two-dimensional real plane [10]. Further efforts of mathematicians were mainly concentrated on the classification of realizations of low-dimensional Lie algebras. Here we would like to point out the important paper [22], where the special technique of so called megaideals was used to list all inequivalent realizations of Lie algebras up to dimension four by vector fields on an arbitrary real (resp. 
complex) finite-dimensional space. (This paper also contains a quite complete list of references on the discussed problem.) At the same time, a number of researchers classified inequivalent realizations of Lie algebras that are important for theoretical physics. Such classifications were done for the Lie algebras of the Euclidean group $\mathrm{E}(3)$ and the Poincaré group $\mathrm{P}(1,3)$ (see, e.g., [8]). Some important results were also obtained for some infinite series of Lie groups and algebras. For instance, the constructive algorithm of embedding of an arbitrary $\mathbb{Z}$-graded Lie algebra into a Lie algebra of polynomial vector fields over a field of arbitrary characteristic was described in [24]. We also have to mention the review paper [6] in which the author consider the problem on realizations of transitive Lie algebras by formal vector fields.

In the present paper we introduce a method to construct an explicit realization of a finitedimensional Lie algebra by left- and right-invariant vector fields on the associated local Lie group using only the structure constants of the algebra. It is shown that in the second canonical coordinates the problem can be solved just by tools of linear algebra and it is reduced to the computation of matrix inversions and matrix exponentiations. The introduced method allows one to construct only regular realizations of Lie algebras but its possible applications are wider. Indeed, if we can construct the realization of the Lie algebra by left-invariant vector fields in canonical coordinates, then we can list other inequivalent realizations of this algebra by vector fields depending on smaller number of independent variables. This can be done by the classification of inequivalent subalgebras of the initial Lie algebra and by the projection of the left-invariant vector fields on the corresponding spaces of right cosets.

Even more complicated problem is solved below. This is the construction of the composition function of a local Lie group whose Lie algebra is known. We emphasize this problem since knowing the composition function gives the complete description of the group structure. Modern approaches to the computation of composition functions is reviewed in the next section, but the main result of this paper can be announced here: The composition function in the second canonical coordinates can be found by quadratures. In Section 5 it is shown that the transition from the second canonical coordinates to the first canonical coordinates can be found by quadratures too. Therefore, if one knows the composition function in any system of canonical coordinates, then the transition to another system of canonical coordinates can be done using special techniques described in this paper.

\section{Preliminary information on theory of Lie groups and algebras}

To make the presentation self-contained and to fix the notations we present basic facts of the Lie theory.

Let $G_{e}$ be an open neighborhood of the identity element $e$ of an $n$-dimensional simply connected real Lie group $G$ that is diffeomorphic to an open subset $U$ of the Euclidean space $\mathbb{R}^{n}$ and let $\psi$ be a mapping realizing this diffeomorphism, $\psi: G_{e} \rightarrow U$. Any group element from the domain $G_{e}$ is uniquely defined by its coordinates. Explicitly this can be expressed as $g_{x}=\psi^{-1}(x) \in G_{e}$, where $x=\left(x^{1}, \ldots, x^{n}\right) \in U$. Therefore, the multiplication rule is represented as ${ }^{1}$

$$
g_{x} g_{y}=g_{z}, \quad z^{i}=\Phi^{i}(x, y), \quad g_{x}, g_{y}, g_{z} \in G_{e} .
$$

The $n$-dimensional vector function $\Phi(x, y)=\left(\Phi^{1}(x, y), \ldots, \Phi^{n}(x, y)\right)$ is called a composition function of the group $G$. Since the group multiplication is associative, the function $\Phi(x, y)$ satisfies the identity

$$
\Phi(x, \Phi(y, z))=\Phi(\Phi(x, y), z) .
$$

\footnotetext{
${ }^{1}$ It is clear that for all objects to be well defined in (2.1), we should consider only pairs of $x$ and $y$ with $g_{x} g_{y} \in G_{e}$. In what follows we omit similar conditions for coordinates.
} 
Without loss of generality one can assume that the zero value of the coordinate tuple corresponds to the identity element $e$ of the group, $\psi(e)=0$. Then the composition function satisfies the initial conditions

$$
\Phi(0, y)=y, \quad \Phi(x, 0)=x .
$$

Denote by $\kappa(x)$ the coordinates of the inverse of $g_{x}$, so $g_{x}^{-1}=g_{\kappa(x)}$. Then the following equalities are obvious:

$$
\Phi(\kappa(x), x)=\Phi(x, \kappa(x))=0,\left.\quad \frac{\partial \kappa^{i}(x)}{\partial x^{j}}\right|_{x=0}=-\delta_{j}^{i} .
$$

The tangent vectors $\partial_{x^{i}}$ at a point $x$ constitute a basis of the tangent space $T_{x} \mathbb{R}^{n}$. The corresponding tangent vectors $\left(\psi^{-1}\right)_{*} \partial_{x^{i}} \equiv \partial_{x^{i}} g_{x} \in T_{g_{x}} G$ form a basis of the tangent space of the group $G$ at the point $g_{x}$. If we assume $x=0$ then the tangent vectors $\left.\partial_{x^{i}} g_{x}\right|_{x=0} \equiv e_{i}$ form a basis of the Lie algebra $\mathfrak{g}$ of the Lie group $G$ with commutation relations

$$
\left[e_{i}, e_{j}\right]=C_{i j}^{k} e_{k}
$$

The numbers $C_{i j}^{k}$ are the structure constants of the Lie algebra $\mathfrak{g}$ in the basis chosen. Hereafter, we follow the Einstein summation convention assuming summation over the repeated indices unless otherwise stated.

The group $G$ acts on itself by the right $R_{g_{y}}$ and the left $L_{g_{y}}$ translations,

$$
R_{g_{y}} g_{x}=g_{x} g_{y}, \quad L_{g_{y}} g_{x}=g_{y} g_{x}
$$

These actions generate the right $T^{R}$ and the left $T^{L}$ regular representations of the group $G$,

$$
T^{R}\left(g_{y}\right) f\left(g_{x}\right)=f\left(g_{x} g_{y}\right), \quad T^{L}\left(g_{y}\right) f\left(g_{x}\right)=f\left(g_{y}^{-1} g_{x}\right), \quad f \in C^{\infty}(G),
$$

whose generators are left- and right-invariant vector fields, respectively,

$$
\begin{aligned}
& \xi_{i}\left(g_{x}\right)=\left(L_{g_{x}}\right)_{*} e_{i} \in T_{g_{x}} G, \quad \psi_{*} \xi_{i}\left(g_{x}\right) \equiv \xi_{i}(x)=\xi_{i}^{j}(x) \frac{\partial}{\partial x^{j}} \\
& \eta_{i}\left(g_{x}\right)=-\left(R_{g_{x}}\right)_{*} e_{i} \in T_{g_{x}} G, \quad \psi_{*} \eta_{i}\left(g_{x}\right) \equiv \eta_{i}(x)=\eta_{i}^{j}(x) \frac{\partial}{\partial x^{j}}, \\
& \xi_{i}^{j}(x)=\left.\frac{\partial \Phi^{j}(x, y)}{\partial y^{i}}\right|_{y=0}, \quad \eta_{i}^{j}(x)=\left.\frac{\partial \Phi^{j}(\kappa(y), x)}{\partial y^{i}}\right|_{y=0}=-\left.\frac{\partial \Phi^{j}(y, x)}{\partial y^{i}}\right|_{y=0} .
\end{aligned}
$$

By $\|\xi(x)\|$ and $\|\eta(x)\|$ we denote the matrices formed by the components $\xi_{i}^{j}(x)$ and $\eta_{i}^{j}(x)$, respectively. The left- and right-invariant basis vector fields $\xi_{i}$ and $\eta_{j}$ satisfy the commutation relations (2.3) and commute with each other, $\left[\xi_{i}, \eta_{j}\right]=0$.

Denote by $\omega^{i}$ and $\sigma^{i}$ the differential 1 -forms on $G$ that are dual to the vector fields $\xi_{i}\left(g_{x}\right)$ and $\eta_{i}\left(g_{x}\right)$, respectively, $\left\langle\omega^{i}, \xi_{j}\right\rangle=\left\langle\sigma^{i}, \eta_{j}\right\rangle=\delta_{j}^{i}$. The basis 1 -forms can be written in coordinates $x^{i}$ as

$$
\omega^{i}=\omega_{j}^{i}(x) d x^{j}, \quad \omega_{j}^{i}(x)=\left\|\xi^{-1}(x)\right\|_{j}^{i}, \quad \sigma^{i}=\sigma_{j}^{i}(x) d x^{j}, \quad \sigma_{j}^{i}(x)=\left\|\eta^{-1}(x)\right\|_{j}^{i} .
$$

The commutation relations (2.3) can be rewritten in the form of equations for the invariant 1 -forms

$$
d \omega^{i}=-\frac{1}{2} C_{j k}^{i} \omega^{j} \wedge \omega^{k}, \quad d \sigma^{i}=-\frac{1}{2} C_{j k}^{i} \sigma^{j} \wedge \sigma^{k} .
$$

These equations are known as Maurer-Cartan equations. 
An element $g$ of the group $G$ generates the inner automorphism $\operatorname{Ad}_{g}$ of the algebra $\mathfrak{g}$,

$$
\operatorname{Ad}_{g} e_{i} \equiv\left(L_{g}\right)_{*}\left(R_{g^{-1}}\right)_{*} e_{i}=\left\|\operatorname{Ad}_{g}\right\|_{i}^{j} e_{j},\left.\quad \frac{\partial}{\partial x^{k}}\left\|\operatorname{Ad}_{g_{x}}\right\|_{i}^{j}\right|_{x=0}=C_{k i}^{j}=\left\|\operatorname{ad} e_{k}\right\|_{i}^{j} .
$$

The matrix $\operatorname{Ad}_{g_{x}}$ can be expressed in terms of the components of basis invariant vector fields and dual 1-forms

$$
\left\|\operatorname{Ad}_{g_{x}}\right\|_{j}^{i}=-\sigma_{k}^{i}(x) \xi_{j}^{k}(x),
$$

so that $\operatorname{Ad}_{g_{x}}=-\|\sigma(x)\| \cdot\|\xi(x)\|$.

The composition function satisfying the initial conditions (2.2) can be uniquely determined from the condition of left- or right-invariance of the vector fields $\xi_{j}$ and $\eta_{j}$, respectively,

$$
\begin{aligned}
& \frac{\partial \Phi^{k}(x, y)}{\partial y^{i}}=\xi_{j}^{k}(\Phi(x, y)) \omega_{i}^{j}(y), \\
& \frac{\partial \Phi^{k}(x, y)}{\partial x^{i}}=\eta_{j}^{k}(\Phi(x, y)) \sigma_{i}^{j}(x) .
\end{aligned}
$$

Note that the above relations hold for any coordinate system on the Lie group $G$. Consider now special kinds of local coordinates.

Let the Lie algebra $\mathfrak{g}$ (as a vector space) be decomposed into a direct sum of subspaces,

$$
\mathfrak{g}=\bigoplus_{k=1}^{m} \mathfrak{g}_{k}=\mathfrak{g}_{1} \oplus \mathfrak{g}_{2} \oplus \cdots \oplus \mathfrak{g}_{m}
$$

We define a mapping $\phi: \mathfrak{g} \rightarrow G$ by $\phi(X)=\prod_{k=1}^{m} \exp \left(X_{k}\right)$ for any $X \in \mathfrak{g}$, where exp: $\mathfrak{g} \rightarrow G$ is the exponential map, $X_{k}$ is the component of $X$ corresponding to $\mathfrak{g}_{k}$ in the decomposition (2.11). There exists a neighborhood $U$ of $0 \in \mathfrak{g}$ such that $\phi$ is a diffeomorphism on a neighborhood $G_{e}$ of the identity element $e \in G$. Therefore, the pair $\left(G_{e}, \phi^{-1}\right)$ is a map on $G$, which is called canonical and the respective local coordinates $x=\left(x^{1}, \ldots, x^{n}\right) \in U$ are called canonical coordinates.

Consider two types of canonical coordinates that are frequently used $[4,5]$. Let the decomposition (2.11) be trivial, i.e., $m=1$ and $\mathfrak{g}_{1}=\mathfrak{g}$. Then

$$
g_{x}=\exp \left(\sum_{i=1}^{n} x^{i} e_{i}\right)=\exp \left(x^{1} e_{1}+\cdots+x^{n} e_{n}\right),
$$

where $e_{1}, \ldots, e_{n}$ constitute a basis of the Lie algebra $\mathfrak{g}$. In this case, the canonical coordinates are called first canonical coordinates. If $m=n$ and thus the subspaces $\mathfrak{g}_{k}$ are necessarily onedimensional, then one has second canonical coordinates,

$$
g_{x}=\prod_{i=1}^{n} \exp \left(x^{i} e_{i}\right)=\exp \left(x^{1} e_{1}\right) \cdots \exp \left(x^{n} e_{n}\right)
$$

(the Einstein summation convention is not implied). The choice of canonical coordinates depends on the problem to be solved.

One of classical problems of the theory of Lie groups is the construction of group multiplication law of a Lie group from the structure constants of the associated Lie algebra. The traditional way consists of two steps in accordance with the well-known Lie theorems. First, the Maurer-Cartan equations (2.6) are to be solved (to be specific we consider the first system), where the components $\omega_{i}^{j}(x)$ of the left-invariant 1 -forms are assumed the unknowns. Note that 
the obvious initial condition $\omega_{i}^{j}(0)=\delta_{i}^{j}$ does not guarantee the solution uniqueness. Therefore, at the first stage one usually chooses and fixes a certain system of canonical coordinates on the Lie group. For instance, the equality $\omega_{j}^{i}(x) x^{j}=x^{i}$ holds true in the first canonical coordinates. Using this equality the solution of (2.6) can be represented in the explicit form [21]

$$
\|\omega(x)\|=\Omega\left(\operatorname{ad}_{x}\right), \quad \Omega(s)=\frac{1-e^{-s}}{s} .
$$

The second step of the computation of the composition function $\Phi(x, y)$ is the integration of the equation (2.9) with the initial condition (2.2).

Another possible way to construct the composition function for a given Lie group from the structure constants of its Lie algebra, requires the explicit computation of the element $Z=$ $\ln \left(e^{X} e^{Y}\right)$ in the form

$$
Z=Y+\int_{0}^{t} \theta\left(e^{t \operatorname{ad}_{X}} e^{\operatorname{ad}_{Y}}\right) X d t
$$

where $\theta(s)=\ln s /(s-1)$, and the elements $X$ and $Y$ belong to a sufficiently small neighborhood of the zero element of the Lie algebra [18]. Indeed, if $X=x^{i} e_{i}$ and $Y=y^{i} e_{i}$ are the decompositions of vectors $X$ and $Y$ in the fixed basis of the Lie algebra $\mathfrak{g}$, respectively, then the components of the vector $Z$ computed by formula (2.13) are the components of the composition function $\Phi(x, y)$ in the first canonical coordinates. One of the consequences of (2.13) is the Baker-Campbell-Hausdorff series which can be obtained by means of the decomposition of the function $\theta(s)$ in power series at the point $s=1$.

Although the presented methods make it possible to find the group multiplication from the commutation relations of the associated Lie algebra, they are not convenient for applications. The first method based on the Lie theorems requires the integration of systems of nonlinear partial differential equations, which appears to be a quite difficult task even for low-dimensional Lie groups. The use of (2.13) involves the complicated calculation of functions depending on matrices as variables (details are discussed below).

Consider a more promising way to construct the composition function. Denote by $\operatorname{Mat}_{m}(\mathbb{R})$ the set of all square $m \times m$ matrices over the field $\mathbb{R}$. Let $\tau: \mathfrak{g} \rightarrow \operatorname{Mat}_{m}(\mathbb{R})$ be a faithful finite-dimensional representation of the Lie algebra $\mathfrak{g}$. Denote the neighborhood of the identity element in the group $G$ as $U \subset G$. Then $V$ will stand for the neighborhood of the zero element of the Lie algebra $\mathfrak{g}$, which is mapped onto $U$ under the action of exponential mapping. Then a mapping $T$ defined as

$$
T(\exp X)=\exp (\tau(X)), \quad X \in V,
$$

gives locally homomorphic mapping of $G$ into $\mathrm{GL}_{m}(\mathbb{R})[4,12]$. It means that there exists such a neighborhood of the identity element $G_{e} \subset U$ that $T\left(g_{1} g_{2}\right)=T\left(g_{1}\right) T\left(g_{2}\right)$ for any $g_{1}, g_{2} \in G_{e}$. Replacing the group elements in (2.1) by their representations, $g \rightarrow T(g)$, results in the matrix equality that can be used for the identification of all components of the composition function $z^{i}=\Phi^{i}(x, y)$; this can be done as far as the representation $\tau$ is faithful. For example, in the first and the second canonical coordinates we get the following matrix equalities (there is no summation over the repeated indices in the second formula)

$$
\begin{aligned}
& \exp \left(\sum_{i=1}^{n} x^{i} \tau\left(e_{i}\right)\right) \exp \left(\sum_{j=1}^{n} y^{j} \tau\left(e_{j}\right)\right)=\exp \left(\sum_{k=1}^{n} z^{k} \tau\left(e_{k}\right)\right), \\
& \prod_{i=1}^{n} \exp \left(x^{i} \tau\left(e_{i}\right)\right) \prod_{j=1}^{n} \exp \left(y^{j} \tau\left(e_{j}\right)\right)=\prod_{k=1}^{n} \exp \left(z^{k} \tau\left(e_{k}\right)\right) .
\end{aligned}
$$


The main disadvantage of the present approach is the absence of a simple procedure that allows to construct a faithful representation of an arbitrary Lie algebra (however some investigations in this direction are in progress [26]).

At the same time, there always exists a special finite-dimensional representation acting in the linear space of the Lie algebra $\mathfrak{g}, \tau=$ ad. In the general case the adjoint representation ad is not faithful since the center of the Lie algebra $\mathfrak{g}$ is a kernel of it, $\mathfrak{z}=\operatorname{ker}$ ad. Let $\left\{e_{\mu}\right\}$ be a basis of $\mathfrak{z}$ and let the set $\left\{e_{a}\right\}$ forms a basis of the subspace $\mathfrak{p}$ complementary to $\mathfrak{z}$. Fix certain canonical coordinates in the local group $G_{e}$ and let these coordinates be connected to the basis $\left\{e_{a}, e_{\mu}\right\}$ of the Lie algebra $\mathfrak{g}$. The functions $\Phi^{a}(x, y)$ can be found from the matrix equality

$$
\operatorname{Ad}_{g_{x}} \operatorname{Ad}_{g_{y}}=\operatorname{Ad}_{g_{z}}, \quad z=\Phi(x, y), \quad \operatorname{Ad}_{e^{X}}=\exp \left(\operatorname{ad}_{X}\right), \quad X \in \mathfrak{g},
$$

and appear to be the components of the composition function for the local quotient group $\bar{G}=G_{e} / \exp (\mathfrak{z})$. So, the problem of construction of the composition function $\Phi=\left(\Phi^{a}, \Phi^{\mu}\right)$ on $G_{e}$ can be reduced to the solution of equations (2.14) with unknown variables $z^{a}=\Phi^{a}(x, y)$ and to the computation of $\Phi^{\mu}(x, y)$ for the central components. The last problem will be solved in Section 4 and it will be shown that functions $\Phi^{\mu}(x, y)$ can be constructed by quadratures.

Finally, we discuss the important issue mentioned in the introduction: How the knowledge of the left- and right-invariant vector fields on a Lie group can be used for the construction of realizations of its Lie algebra by vector fields in finite-dimensional spaces?

Consider an $m$-dimensional space $M$ (an open domain in $\mathbb{R}^{m}$ ) with coordinates $q=\left(q^{1}, \ldots\right.$, $\left.q^{m}\right)$. Let $X_{i}=X_{i}^{a}(q) \partial_{q^{a}}$ be vector fields on $M$ that realize an $n$-dimensional Lie algebra $\mathfrak{g}$. Then there exists one and only one local transformation Lie group $G_{e}$ of $M$ whose Lie algebra coincides with the above realization of $\mathfrak{g}$. Let $U=\psi\left(G_{e}\right) \subset \mathbb{R}^{n}$ be an image of $G_{e}$ under coordinate mapping $\psi$. It means that there exists a function $\Psi: M \times U \rightarrow M$ such that

$$
\begin{aligned}
& \Psi(\Psi(q, x), y)=\Psi(q, \Phi(x, y)), \quad \Psi(q, 0)=q, \quad q \in M, \quad x, y \in U, \\
& X_{i}^{a}(q)=\left.\frac{\partial \Psi^{a}(q, x)}{\partial x^{i}}\right|_{x=0} .
\end{aligned}
$$

The vector fields $X_{i}$ that are defined by (2.15) are called (infinitesimal) generators of the action of the group $G_{e}$ on $M$.

Suppose that the action of $G_{e}$ on $M$ is transitive. It means that any point $q_{0} \in M$ has a neighborhood $V \subset M$ such that for any $q \in V$ there exists an element $g_{x} \in G_{e}$ with $q=$ $\Psi\left(q_{0}, x\right)$. This implies that $\operatorname{rank}\left(X_{i}^{a}(q)\right)=m, q \in V$. We fix a point $q_{0} \in M$ and denote by $H$ the isotropy group of the point $q_{0}$ under the action of $G_{e}, H=\left\{g_{x} \in G_{e} \mid \Psi\left(q_{0}, x\right)=q_{0}\right\}$, which is a subgroup of $G_{e}$. As a result, we obtain a $G_{e}$-equivariant diffeomorphism between points of the space $M$ and elements of the space of right cosets $H \backslash G_{e}$ [11]. The choice of the point $q_{0} \in M$ is not essential as far as the isotropy groups of different points of a homogeneous space are conjugate.

So, the transitive action of local transformation group is defined by the pair $\left(G_{e}, H\right)$, where $G_{e}$ is a local Lie group and $H$ is a subgroup of $G_{e}$. This is equivalent to the assignment of the pair $(\mathfrak{g}, \mathfrak{h})$, where $\mathfrak{g}$ is the Lie algebra of the group $G_{e}$, and $\mathfrak{h}$ is the Lie algebra of the group $H$. Inversely, given a Lie algebra $\mathfrak{g}$ and its subalgebra $\mathfrak{h}$, we can construct the corresponding local groups $G_{e}$ and $H$ and the domain $M$, where $G_{e}$ acts transitively; $M$ can be defined as the space of right cosets $H \backslash G_{e}$. Subalgebras of $\mathfrak{g}$ that are connected by inner automorphisms correspond to equivalent actions of the local group $G_{e}$, because of equivariant diffeomorphism of the homogeneous spaces.

Note that in general case the group action of $G_{e}$ on the space of right cosets $H \backslash G_{e}$ may be not effective, i.e., there may exist $g_{x} \in G_{e}$ such that $\Psi(q, x)=q$ for all $q \in H \backslash G_{e}$. A number of researchers without loss of generality restrict their consideration to the class of effective actions 
of transformation groups. For instance, if the action of $G_{e}$ on $H \backslash G_{e}$ is not effective, then we can consider the effective action of the quotient group $G_{e} / N$ on the given homogeneous space, where $N$ is the maximal normal subgroup of $G_{e}$ that is contained in $H$ [11]. Here we do not restrict ourselves to effective group actions and allow $H$ to contain a nontrivial normal subgroup of $G_{e}$. In terms of Lie algebras, it means that the subalgebra $\mathfrak{h}$ may include nonzero ideals of the algebra $\mathfrak{g}$.

An arbitrary element of the Lie group $G_{e}$ can be represented as $g_{x}=h_{y} \bar{g}_{q}$, where $h_{y} \in H$ and $\bar{g}_{q}$ is a fixed representative of the right coset $H g_{x}$,

$$
x=(q, y), \quad x^{a}=q^{a}, \quad a=1, \ldots, m, \quad x^{m+\beta}=y^{\beta}, \quad \beta=1, \ldots, n-m .
$$

Here $q^{a}$ are coordinates in the space of right cosets $H \backslash G_{e}$ and $y^{\beta}$ the coordinates in the subgroup $H$. Therefore, the action of the local group $G_{e}$ on $M \simeq H \backslash G_{e}$ is reduced to the transformation of the coset representatives $\bar{g}_{q} g_{z}=h(q, z) \bar{g}_{\Psi(q, z)}$, where $h(q, z) \in H$ is a factor of the homogeneous space. Multiplying the last equality by $h_{y}$ we get

$$
g_{\Phi((q, y), z)}=\left(h_{y} \bar{g}_{q}\right) g_{z}=\left(h_{y} h(q, z)\right) \bar{g}_{\Psi(q, z)} .
$$

This implies that each $a$-th component of the composition function $\Phi(x, z)=\Phi((q, y), z)$ does not depend on the coordinates in $H$ and coincides with the respective component of the composition function $\Psi(q, z)$

$$
\Psi^{a}(q, z)=\Phi^{a}((q, y), z), \quad a=1, \ldots, m .
$$

The equalities (2.16), (2.4) and (2.15) allow us to connect the left-invariant vector fields $\xi_{i}$ on $G_{e}$ with the corresponding generators $X_{i}$ of the group action on the homogeneous space $M \simeq H \backslash G_{e}$

$$
\begin{aligned}
& \xi_{i}(q, y)=\xi_{i}^{a}(q) \frac{\partial}{\partial q^{a}}+\xi_{i}^{\beta}(q, y) \frac{\partial}{\partial y^{\beta}}, \\
& X_{i}(q)=\xi_{i}^{a}(q) \frac{\partial}{\partial q^{a}} .
\end{aligned}
$$

Concluding this section, we would like to make the following remark. The problems of the construction of generators of the transitive transformation group and the realization of the Lie algebra by vector fields with a given number of independent variables are connected but definitely are not equivalent. The second problem is much more complicated and requires more sophisticated methods (see, for example, $[16,19,22]$ ). Our paper is concentrated on the solution of the first problem.

\section{Computation of invariant vector fields and 1-forms in second canonical coordinates}

The practical computation of components of invariant vector fields and 1-forms in the first canonical coordinates is a complicated problem even for low-dimensional Lie groups. The application of the formulas (2.12), (2.13) to the explicit computation requires evaluation of the involved functions at the matrices $\operatorname{ad}_{X}$ and $\exp \left(t \operatorname{ad}_{X}\right) \exp \left(\operatorname{ad}_{Y}\right)$. These problems are linear and they are solved by reduction of the matrices to their Jordan normal forms. In the first canonical coordinates the matrices $\operatorname{ad}_{X}$ and $\exp \left(t \operatorname{ad}_{X}\right) \exp \left(\operatorname{ad}_{Y}\right)$ depend on $n$ and $2 n+1$ variables $x^{i}$ and $x^{i}, y^{j}, t$, respectively, which makes the problem quite complicated. If all the above calculations are done, then the result of computation is cumbersome and hardly applicable in practice. In the second canonical coordinates the components of the invariant vector fields 
and 1-forms are relatively simple and can be easily calculated. This fact is proven by the following algorithm, which originates from the work of one of the authors of the present paper [25].

We apply the differential of a left translation $\left(L_{g_{x}}\right)_{*}$ to a basis vector $e_{k}$ of the Lie algebra $\mathfrak{g}$. Then, taking into account the equations (2.4) that define $\xi_{i}^{j}(x)$, we get

$$
\begin{aligned}
\left(L_{g_{x}}\right)_{*} e_{k} & =\left.\left(L_{g_{x}}\right)_{*} \partial_{y^{k}} g_{y}\right|_{y=0}=\left.\partial_{y^{k}}\left(g_{x} g_{y}\right)\right|_{y=0}=\left.\partial_{y^{k}} g_{\Phi(x, y)}\right|_{y=0} \\
& =\left.\frac{\partial \Phi^{i}(x, y)}{\partial y^{k}}\right|_{y=0} \partial_{x^{i}} g_{x}=\xi_{k}^{i}(x) \partial_{x^{i}} g_{x}
\end{aligned}
$$

which is equivalent to the conditions

$$
\omega_{k}^{i}(x) e_{i}=\left(L_{g_{x}^{-1}}\right)_{*} \partial_{x^{k}} g_{x} .
$$

Choose the second canonical coordinates on the local group $G_{e}$

$$
g_{x}=g_{n}\left(x^{n}\right) \cdots g_{1}\left(x^{1}\right), \quad g_{i}(t) \equiv \exp \left(t e_{i}\right)
$$

The relation $\left.\partial_{t} g_{k}(t)\right|_{t=0}=e_{k}$ obviously implies $\partial_{x^{1}} g_{x}=\left(L_{g_{x}}\right)_{*} e_{1}$. For any $k>1$ we obtain $\partial_{x^{k}} g_{x}=\left(L_{g_{n}}\right)_{*} \cdots\left(L_{g_{k}}\right)_{*}\left(R_{g_{1}}\right)_{*} \cdots\left(R_{g_{k-1}}\right)_{*} e_{k}$. In the chosen coordinate system we also have

$$
\left(L_{g_{x}^{-1}}\right)_{*}=\left(L_{g_{1}^{-1}}\right)_{*}\left(L_{g_{2}^{-1}}\right)_{*} \cdots\left(L_{g_{n}^{-1}}\right)_{*} .
$$

Due to the commutativity of the right and left translations and in view of (2.7), the conditions (3.1) can be rewritten as

$$
\begin{aligned}
\omega_{k}^{i}(x) e_{i} & =\left[\left(L_{g_{1}^{-1}}\right)_{*}\left(R_{g_{1}}\right)_{*}\right]\left[\left(L_{g_{2}^{-1}}\right)_{*}\left(R_{g_{2}}\right)_{*}\right] \cdots\left[\left(L_{g_{k-1}^{-1}}\right)_{*}\left(R_{g_{k-1}}\right)_{*}\right] e_{k} \\
& =\operatorname{Ad}_{g_{1}^{-1}} \operatorname{Ad}_{g_{2}^{-1}} \cdots \operatorname{Ad}_{g_{k-1}^{-1}} e_{k} .
\end{aligned}
$$

So, the components of left-invariant 1-forms in the second canonical coordinates are calculated by the formulas

$$
\begin{aligned}
& \omega_{1}^{i}(x)=\delta_{1}^{i}, \\
& \omega_{k}^{i}(x)=\| \exp \left(-x^{1} \text { ad } e_{1}\right) \exp \left(-x^{2} \text { ad } e_{2}\right) \cdots \exp \left(-x^{k-1} \text { ad } e_{k-1}\right) \|_{k}^{i}, \quad k>1 .
\end{aligned}
$$

The use of (2.5) allows us to find the components of right-invariant 1-forms and left- and right-invariant vector fields. In view of (3.3), the general structure of the left-invariant 1-forms in the chosen coordinates is

$$
\omega^{i}(x)=\delta_{1}^{i} d x^{1}+\omega_{2}^{i}\left(x^{1}\right) d x^{2}+\omega_{3}^{i}\left(x^{1}, x^{2}\right) d x^{3}+\cdots+\omega_{n}^{i}\left(x^{1}, \ldots, x^{n-1}\right) d x^{n} .
$$

It's obvious that $\xi_{1}=\partial_{x^{1}}$ and, if $\left[e_{1}, e_{2}\right]=0$, then also $\xi_{2}=\partial_{x^{2}}$, etc. All the functions $\xi_{i}^{j}$ do not depend on $x^{n}$ and, if the condition $\left[e_{n}, e_{n-1}\right]=0$ is satisfied, then $\xi_{i}^{j}$ do also not depend on $x^{n-1}$, etc.

The suggested method of the construction of invariant fields and 1-forms can be easily generalized for an arbitrary coordinate system of the second type. For instance, one can choose an arbitrary order of exponentials in (3.2): $g_{x}=g_{\pi(n)}\left(x^{\pi(n)}\right) \cdots g_{\pi(1)}\left(x^{\pi(1)}\right)$, where $\pi \in S_{n}$ is a certain permutation of the set $\{1, \ldots, n\}$. In this case the left-invariant field $\xi_{\pi(1)}$ is diagonal $\xi_{\pi(1)}=\partial_{x^{\pi(1)}}$. Therefore, changing the basis of the Lie algebra we can diagonalize any given vector field along the chosen direction.

The second canonical coordinates are especially convenient for the coordinate realization of generators of the group action on homogeneous space. Indeed, let $M$ be the right homogeneous 
space equivariant to the space of right cosets $M \simeq H \backslash G_{e}$, let $\mathfrak{h}$ be the Lie algebra of group $H$ with a basis $\left\{e_{\beta}\right\}$ and let $\mathfrak{p}=\left\{e_{a}\right\}$ be a linear subspace complementary to the space $\mathfrak{h}$. We choose the second canonical coordinates on $G_{e}$ with

$$
g_{(q, y)}=\prod_{\beta=1}^{\operatorname{dim} \mathfrak{h}} \exp \left(y^{\beta} e_{\beta}\right) \prod_{a=1}^{\operatorname{dim} \mathfrak{p}} \exp \left(q^{a} e_{a}\right)
$$

Then the coordinate form of left-invariant vector fields and generators of the transformation group is given by (2.17) and (2.18), respectively.

We should emphasize that a researcher who solves the problem of realization of a Lie algebra by left-invariant vector fields on the associated Lie group can take into account only the commutation relations. For instance, one can assume $\xi_{1}=\partial_{x^{1}}$ and also can choose the diagonal form for all the fields commuting with it. After that, the system of constructed vector fields is to be completed by the rest of them with unknown coefficients; as a result, the overdetermined system of differential equations is to be obtained from the commutation relations. Consequent integration of this system gives the solution of the problem. This procedure provides vector fields, which will coincide with the left-invariant vector fields in the second canonical coordinates (up to coordinate transformations and up to a basis change from the automorphism group). In other words, the simplest realization of a Lie algebra by left-invariant vector fields is a realization in the second canonical coordinates and in that sense, this type of coordinates is privileged.

As an example, consider the six-dimensional unsolvable Lie algebra $\mathfrak{g}$ with the following non-zero commutation relations:

$$
\begin{array}{llrl}
{\left[e_{1}, e_{2}\right]=e_{6},} & {\left[e_{1}, e_{4}\right]=-e_{1},} & {\left[e_{1}, e_{5}\right]=e_{2},} & {\left[e_{2}, e_{3}\right]=e_{1},} \\
{\left[e_{2}, e_{4}\right]=e_{2},} & {\left[e_{3}, e_{4}\right]=-2 e_{3}, \quad\left[e_{3}, e_{5}\right]=e_{4}, \quad\left[e_{4}, e_{5}\right]=-2 e_{5} .}
\end{array}
$$

This algebra is a semidirect sum of the three-dimensional nilpotent ideal and the simple Lie algebra $\mathfrak{s o}(1,2)$.

We choose the second canonical coordinates $(3.2) g_{x}=g_{6}\left(x^{6}\right) g_{5}\left(x^{5}\right) \cdots g_{1}\left(x^{1}\right)$ on the corresponding local Lie group. The coordinate representations of basic left-invariant 1-forms are obtained by the computation of matrix exponentials $\exp \left(-x^{i}\right.$ ad $\left.e_{i}\right)$ according to (3.3),

$$
\begin{aligned}
& \omega^{1}=d x^{1}-x^{2} d x^{3}+\left(x^{1}-2 x^{2} x^{3}\right) d x^{4}+x^{3} e^{2 x^{4}}\left(x^{2} x^{3}-x^{1}\right) d x^{5}, \\
& \omega^{2}=d x^{2}-x^{2} d x^{4}+e^{2 x^{4}}\left(x^{2} x^{3}-x^{1}\right) d x^{5}, \\
& \omega^{3}=d x^{3}+2 x^{3} d x^{4}-\left(x^{3}\right)^{2} e^{2 x^{4}} d x^{5}, \\
& \omega^{4}=d x^{4}-x^{3} e^{2 x^{4}} d x^{5}, \\
& \omega^{5}=e^{2 x^{4}} d x^{5} \\
& \omega^{6}=d x^{6}-x^{1} d x^{2}-\frac{1}{2}\left(x^{2}\right)^{2} d x^{3}+x^{2}\left(x^{1}-x^{2} x^{3}\right) d x^{4}+\frac{1}{2} e^{2 x^{4}}\left(x^{1}-x^{2} x^{3}\right)^{2} d x^{5} .
\end{aligned}
$$

The matrices $\operatorname{Ad}_{g_{x}^{-1}}$ and their inverses $\operatorname{Ad}_{g_{x}}$ are constructed via the multiplication of the matrix exponentials $\exp \left(-x^{i}\right.$ ad $\left.e_{i}\right)$ in the appropriate order, here - in the decreasing order of indices. Components of the right-invariant 1-forms are given by the formula $\|\sigma(x)\|=-\operatorname{Ad}_{g_{x}} \cdot\|\omega(x)\|$ as it follows from (2.8). The matrices of components of the right- and left-invariant vector fields are computed as the inverses of the corresponding matrices for 1-forms $\|\sigma(x)\|$ and $\|\omega(x)\|$, respectively. In this example, the final expression for the left- and right-invariant vector fields 
looks as follows (the notation $\partial_{x^{i}} \equiv \partial / \partial x^{i}$ is assumed):

$$
\begin{aligned}
& \xi_{1}=\partial_{x^{1}}, \quad \xi_{2}=\partial_{x^{2}}+x^{1} \partial_{x^{6}}, \quad \xi_{3}=x^{2} \partial_{x^{1}}+\partial_{x^{3}}+\frac{1}{2}\left(x^{2}\right)^{2} \partial_{x^{6}}, \\
& \xi_{4}=-x^{1} \partial_{x^{1}}+x^{2} \partial_{x^{2}}-2 x^{3} \partial_{x^{3}}+\partial_{x^{4}}, \\
& \xi_{5}=x^{1} \partial_{x^{2}}-\left(x^{3}\right)^{2} \partial_{x^{3}}+x^{3} \partial_{x^{4}}+e^{-2 x^{4}} \partial_{x^{5}}+\frac{1}{2}\left(x^{1}\right)^{2} \partial_{x^{6}}, \quad \xi_{6}=\partial_{x^{6}}, \\
& \eta_{1}=-\left(e^{-x^{4}}+x^{3} x^{5} e^{x^{4}}\right) \partial_{x^{1}}-x^{5} e^{x^{4}} \partial_{x^{2}}-x^{2}\left(e^{-x^{4}}+x^{3} x^{5} e^{x^{4}}\right) \partial_{x^{6}}, \\
& \eta_{2}=-e^{x^{4}}\left(x^{3} \partial_{x^{1}}+\partial_{x^{2}}+x^{2} x^{3} \partial_{x^{6}}\right), \quad \eta_{3}=-e^{-2 x^{4}} \partial_{x^{3}}-x^{5} \partial_{x^{4}}+\left(x^{5}\right)^{2} \partial_{x^{5}}, \\
& \eta_{4}=-\partial_{x^{4}}+2 x^{5} \partial_{x^{5}}, \quad \eta_{5}=-\partial_{x^{5}}, \quad \eta_{6}=-\partial_{x^{6}} .
\end{aligned}
$$

Consider the four-dimensional homogeneous space $M=H \backslash G_{e}$ with the isotropy subalgebra $\mathfrak{h}=\left\{e_{4}, e_{5}\right\}, H=\exp (\mathfrak{h})$. Since the basis vector $e_{6}$ generates the center of $\mathfrak{g}$, the element $g_{6}\left(x^{6}\right)$ commutes with any element of the group,

$$
g_{6}\left(x^{6}\right) g_{5}\left(x^{5}\right) \cdots g_{1}\left(x^{1}\right)=g_{5}\left(x^{5}\right) \cdots g_{1}\left(x^{1}\right) g_{6}\left(x^{6}\right) .
$$

Therefore, the element $g_{x}$ has representation (3.4) in the chosen second canonical coordinates and the generators $X_{i}$ of the transformation group of homogeneous space with the local coordinates $q^{1}=x^{1}, q^{2}=x^{2}, q^{3}=x^{3}, q^{4}=x^{6}$ are obtained from the left-invariant fields (3.7) by the formal substitution $\partial_{x^{1}} \rightarrow \partial_{q^{1}}, \partial_{x^{2}} \rightarrow \partial_{q^{2}}, \partial_{x^{3}} \rightarrow \partial_{q^{3}}, \partial_{x^{4}} \rightarrow 0, \partial_{x^{5}} \rightarrow 0$ and $\partial_{x^{6}} \rightarrow \partial_{q^{4}}$

$$
\begin{aligned}
& X_{1}=\partial_{q^{1}}, \quad X_{2}=\partial_{q^{2}}+q^{1} \partial_{q^{4}}, \quad X_{3}=q^{2} \partial_{q^{1}}+\partial_{q^{3}}+\frac{1}{2}\left(q^{2}\right)^{2} \partial_{q^{4}}, \\
& X_{4}=-q^{1} \partial_{q^{1}}+q^{2} \partial_{q^{2}}-2 q^{3} \partial_{q^{3}}, \quad X_{5}=q^{1} \partial_{q^{2}}-\left(q^{3}\right)^{2} \partial_{q^{3}}+\frac{1}{2}\left(q^{1}\right)^{2} \partial_{q^{4}}, \quad X_{6}=\partial_{q^{4}} .
\end{aligned}
$$

The problem on realizations of a Lie algebra whose commutation relations contain arbitrary parameters is quite common in applications. Isotropy subalgebras may also depend on arbitrary parameters. The described method is still useful in these cases. We illustrate this by a simple example.

The canonical basis of the Poincaré algebra $\mathfrak{p}(1,3)$ is $\left\{P_{A}, J_{A B}, A<B\right\}$, where $P_{A}$ are generators of translations and $J_{A B}$ are generators of Lorentz transformations in the Minkowski spacetime, $A, B=0,1,2,3$. The complete classification of all inequivalent subalgebras of the algebra $\mathfrak{p}(1,3)$ is given in [7]. Consider the four-dimensional subalgebra $\mathfrak{g}$ from this classification with the basis elements

$$
e_{1}=P_{1}, \quad e_{2}=P_{2}, \quad e_{3}=J_{12}+\alpha J_{03}, \quad e_{4}=P_{0}+P_{3}, \quad \alpha \in \mathbb{R},
$$

which satisfy the following nonzero commutation relations:

$$
\left[e_{1}, e_{3}\right]=e_{2}, \quad\left[e_{2}, e_{3}\right]=-e_{1}, \quad\left[e_{3}, e_{4}\right]=-\alpha e_{4}
$$

We construct a realization of the algebra $\mathfrak{g}$ that is associated with the isotropy subalgebra $\mathfrak{h}$ spanned by the element $\left\{e_{3}+b e_{4}\right\}$.

For this purpose, we choose the second canonical coordinates $\left(y, q^{1}, q^{2}, q^{3}\right)$ on a neighborhood of the identity in the Lie group with the Lie algebra $\mathfrak{g}$ such that an arbitrary element from this neighborhood is represented as

$$
g=\exp \left(y\left(e_{3}+b e_{4}\right)\right) \exp \left(q^{3} e_{3}\right) \exp \left(q^{2} e_{2}\right) \exp \left(q^{1} e_{1}\right), \quad b \in \mathbb{R} .
$$


The matrix $\mathrm{Ad}_{g}$ is a result of matrix exponentiations. The components of left-invariant 1-forms $\omega_{j}^{i}$ are to be found on the next step and the formulas (2.5) and (2.8) give expressions for leftand right-invariant vector fields in the considered coordinate system $\left(\partial_{y} \equiv \partial / \partial y, \partial_{q^{i}} \equiv \partial / \partial q^{i}\right)$

$$
\begin{aligned}
& \xi_{1}=\partial_{q^{1}}, \quad \xi_{2}=\partial_{q^{2}}, \quad \xi_{3}=-q^{2} \partial_{q^{1}}+q^{1} \partial_{q^{2}}+\partial_{q^{3}}, \quad \xi_{4}=(1 / b) e^{-\alpha q^{3}}\left(\partial_{y}-\partial_{q^{3}}\right), \\
& \eta_{1}=-\cos \left(y+q^{3}\right) \partial_{q^{1}}-\sin \left(y+q^{3}\right) \partial_{q^{2}}, \quad \eta_{2}=\sin \left(y+q^{3}\right) \partial_{q^{1}}-\cos \left(y+q^{3}\right) \partial_{q^{2}}, \\
& \eta_{3}=\left(e^{\alpha y}-1\right) \partial_{y}-e^{\alpha y} \partial_{q^{3}}, \quad \eta_{4}=(1 / b) e^{\alpha y}\left(\partial_{q^{3}}-\partial_{y}\right) .
\end{aligned}
$$

Finally, we can construct the generators of the transformation group whose isotropy subgroup is associated with the subalgebra $\mathfrak{h}=\left\{e_{3}+b e_{4}\right\}$. As mentioned above, this can be realized by the restriction of the left-invariant vector fields on the space of functions that do not depend on the variable $y$. This allows us to formally substitute $\partial_{y} \rightarrow 0$ and get

$$
X_{1}=\partial_{q^{1}}, \quad X_{2}=\partial_{q^{2}}, \quad X_{3}=-q^{2} \partial_{q^{1}}+q^{1} \partial_{q^{2}}+\partial_{q^{3}}, \quad X_{4}=-(1 / b) e^{-\alpha q^{3}} \partial_{q^{3}} .
$$

\section{Composition function in second canonical coordinates}

We represent the Lie algebra $\mathfrak{g}$ as the direct sum of two subspaces - the center $\mathfrak{z}=$ ker ad of $\mathfrak{g}$ and a linear complement $\mathfrak{p}$ to $\mathfrak{z}$ in $\mathfrak{g}, \mathfrak{g}=\mathfrak{z} \oplus \mathfrak{p}$. Let $\left\{e_{\mu}\right\}$ and $\left\{e_{a}\right\}$ be bases in $\mathfrak{z}$ and in $\mathfrak{p}$, respectively. Since any element of $\exp (\mathfrak{z})$ commutes with any element of the local group $G_{e}$, in the second canonical coordinates we get (the Einstein summation convention is not assumed)

$$
\begin{aligned}
g_{x} g_{y} & =\left(\prod_{\mu=1}^{\operatorname{dim} \mathfrak{z}} \exp \left(x^{\mu} e_{\mu}\right) \prod_{a=1}^{\operatorname{dim} \mathfrak{p}} \exp \left(x^{a} e_{a}\right)\right)\left(\prod_{\nu=1}^{\operatorname{dim} \mathfrak{z}} \exp \left(y^{\nu} e_{\nu}\right) \prod_{b=1}^{\operatorname{dim} \mathfrak{p}} \exp \left(y^{b} e_{b}\right)\right) \\
& =\prod_{\mu=1}^{\operatorname{dim} \mathfrak{z}} \exp \left(\left(x^{\mu}+y^{\mu}\right) e_{\mu}\right)\left(\prod_{a=1}^{\operatorname{dim} \mathfrak{p}} \exp \left(x^{a} e_{a}\right) \prod_{b=1}^{\operatorname{dim} \mathfrak{p}} \exp \left(y^{b} e_{b}\right)\right) .
\end{aligned}
$$

In the general case, the subspace $\mathfrak{p}$ is not a subalgebra of $\mathfrak{g}$ and the expression in the last big brackets can be rewritten as

$$
\prod_{a=1}^{\operatorname{dim} \mathfrak{p}} \exp \left(x^{a} e_{a}\right) \prod_{b=1}^{\operatorname{dim} \mathfrak{p}} \exp \left(y^{b} e_{b}\right)=\prod_{\mu=1}^{\operatorname{dim} \mathfrak{z}} \exp \left(\Theta^{\mu}(x, y) e_{\mu}\right) \prod_{a=1}^{\operatorname{dim} \mathfrak{p}} \exp \left(\bar{\Phi}^{a}(x, y) e_{a}\right) .
$$

The equality (4.2) should be considered as the definition of the functions $\Theta^{\mu}(x, y)$ and $\bar{\Phi}^{a}(x, y)$. It is important that these functions depend only on coordinates $x^{a}$ and $y^{a}$ corresponding to the subspace $\mathfrak{p}$. The substitution of (4.2) into (4.1) gives

$$
g_{x} g_{y}=\prod_{\mu=1}^{\operatorname{dim} \mathfrak{z}} \exp \left(\left(x^{\mu}+y^{\mu}+\Theta^{\mu}(x, y)\right) e_{\mu}\right) \prod_{a=1}^{\operatorname{dim} \mathfrak{p}} \exp \left(\bar{\Phi}^{a}(x, y) e_{a}\right) .
$$

Therefore, the composition function $\Phi(x, y)$ in the second canonical coordinates related to the decomposition $\mathfrak{g}=\mathfrak{z} \oplus \mathfrak{p}$ looks as

$$
\Phi^{\mu}(x, y)=x^{\mu}+y^{\mu}+\Theta^{\mu}(x, y), \quad \Phi^{a}(x, y)=\bar{\Phi}^{a}(x, y) .
$$

It is necessary to note here that the subspace $\mathfrak{p}$ in the decomposition $\mathfrak{g}=\mathfrak{z} \oplus \mathfrak{p}$ can be chosen in many different ways. This ambiguity originates from the possibility to change the basis of the Lie algebra $\mathfrak{g}: e_{\mu} \rightarrow e_{\mu}, e_{a} \rightarrow e_{a}+\lambda_{a}^{\mu} e_{\mu}$, where $\left(\lambda_{a}^{\mu}\right)$ is an arbitrary matrix of the appropriate size. It is easy to prove that this basis change makes the functions $\Theta^{\mu}$ and $\bar{\Phi}^{a}$ to transform as

$$
\Theta^{\mu}(x, y) \rightarrow \Theta^{\mu}(x, y)+\left(x^{a}+y^{a}-\bar{\Phi}^{a}(x, y)\right) \lambda_{a}^{\mu}, \quad \bar{\Phi}^{a}(x, y) \rightarrow \bar{\Phi}^{a}(x, y) .
$$


Proposition 1. The composition function for a local Lie group $G_{e}$ in the second canonical coordinates can be found by quadratures.

Proof. The proposition is proven in a constructive way by the demonstration of the algorithm for computing the functions $\bar{\Phi}^{a}(x, y)$ and $\Theta^{\mu}(x, y)$ that are involved in the representation (4.3), for the composition function of local group $G_{e}$.

Since ad $e_{\mu}=0$, the matrix of the adjoint representation $\operatorname{Ad}_{g_{x}}$ in the second canonical coordinates depends only on $x^{a}$, and hence

$$
\operatorname{Ad}_{g_{x}}=\prod_{a=1}^{\operatorname{dim} \mathfrak{p}} \exp \left(x^{a} \operatorname{ad} e_{a}\right)
$$

Then from (2.14) and (4.3) we obtain

$$
\prod_{a=1}^{\operatorname{dim} \mathfrak{p}} \exp \left(x^{a} \operatorname{ad} e_{a}\right) \prod_{b=1}^{\operatorname{dim} \mathfrak{p}} \exp \left(y^{b} \operatorname{ad} e_{b}\right)=\prod_{a=1}^{\operatorname{dim} \mathfrak{p}} \exp \left(\bar{\Phi}^{a}(x, y) \operatorname{ad} e_{a}\right)
$$

Let a tuple of $x^{a}$ be the coordinates in the local quotient group $G_{e} / \exp (\mathfrak{z})$. Then the matrices (4.4) are the matrices of adjoint representation of the group $G_{e}$ and, at the same time, they form a faithful representation of the quotient group $G_{e} / \exp (\mathfrak{z})$ that acts in the linear space $\mathfrak{g}$. Therefore, the matrix relation (4.5) allows us to define uniquely the functions $\bar{\Phi}^{a}(x, y)$, which are the composition functions for the local quotient group $G_{e} / \exp (\mathfrak{z})$. So, the problem (4.3) is reduced to finding the still undefined functions $\Theta^{\mu}(x, y)$.

Let $\xi_{i}(x)=\xi_{i}^{j}(x) \partial_{x^{j}}$ be the left-invariant vector fields on the group $G_{e}$ which are written in the second canonical coordinates, and let $\omega^{i}(x)=\omega_{j}^{i}(x) d x^{j}$ be the corresponding left-invariant 1 -forms. As shown in the previous section, in given coordinates this is a problem of linear algebra and can be solved by the computation of matrix exponentials. Note that the components $\xi_{i}^{j}(x)$ and $\omega_{j}^{i}(x)$ are functions only of the coordinates $x^{a}$ and do not depend on the coordinates of the center $\exp (\mathfrak{z})$ (see (4.3) for the composition function of the local group $G_{e}$ ).

Setting $i=a, k=\mu$ in (2.10) and then taking into account (4.3), we obtain a system of differential equations for the unknown functions $\Theta^{\mu}(x, y)$, where coordinates $x^{a}$ are parameters,

$$
\frac{\partial \Theta^{\mu}(x, y)}{\partial y^{a}}=\xi_{j}^{\mu}(\bar{\Phi}(x, y)) \omega_{a}^{j}(y)
$$

The system (4.6) is completely integrable since the 1 -forms $\xi_{j}^{\mu}(\bar{\Phi}(x, y)) \omega_{a}^{j}(y) d y^{a}$ are closed and the solution of this system with the initial condition $\Theta^{\mu}(x, 0)=0$ is given by the integral

$$
\Theta^{\mu}(x, y)=\int_{0}^{y} \xi_{j}^{\mu}(\bar{\Phi}(x, z)) \omega_{a}^{j}(z) d z^{a}
$$

The proposition is proven.

In order to illustrate results of this section, we construct an example of the composition function for the local Lie group $G_{e}$ that corresponds to the six-dimensional Lie algebra $\mathfrak{g}$ defined by the commutation relations (3.5). The center $\mathfrak{z}$ of $\mathfrak{g}$ is one-dimensional, $\mathfrak{z}=\left\{e_{6}\right\}$. Let the five-dimensional subspace $\mathfrak{p}=\left\{e_{1}, e_{2}, e_{3}, e_{4}, e_{5}\right\}$ be the chosen linear complement to $\mathfrak{z}$.

We compute the matrix exponentials $\exp \left(x^{i}\right.$ ad $\left.e_{i}\right)$ and substitute them into the matrix equality (4.5), which gives a system of algebraic equations on the unknown functions $\bar{\Phi}^{a}(x, y)$. Solving 
this system we get

$$
\begin{aligned}
& \Phi^{1}(x, y)=\bar{\Phi}^{1}(x, y)=x^{1} e^{-y^{4}}+y^{1}+y^{3} e^{y^{4}}\left(x^{2}+x^{1} y^{5}\right) \\
& \Phi^{2}(x, y)=\bar{\Phi}^{2}(x, y)=e^{y^{4}}\left(x^{2}+x^{1} y^{5}\right)+y^{2} \\
& \Phi^{3}(x, y)=\bar{\Phi}^{3}(x, y)=\frac{e^{-2 y^{4}} x^{3}+y^{3}\left(1+x^{3} y^{5}\right)}{1+x^{3} y^{5}} \\
& \Phi^{4}(x, y)=\bar{\Phi}^{4}(x, y)=x^{4}+y^{4}+\ln \left(1+x^{3} y^{5}\right) \\
& \Phi_{5}(x, y)=\bar{\Phi}^{5}(x, y)=\frac{x^{5}+e^{-2 x^{4}} y^{5}+x^{3} x^{5} y^{5}}{1+x^{3} y^{5}}
\end{aligned}
$$

We substitute the expressions for left-invariant vector fields $\xi_{i}(x)$, dual 1-forms $\omega^{i}(x)$ and functions that are presented in (3.7), (3.6) and (4.8), respectively, into (4.7). The computing the resulting integral jointly with (4.3) gives the sixth component of the composition function

$$
\Phi^{6}(x, y)=x^{6}+y^{6}+\frac{1}{2}\left(x^{1}\right)^{2} y^{5}+y^{2} y^{3} e^{y^{4}}\left(x^{1} y^{5}+x^{2}\right)+x^{1} y^{2} e^{-y^{4}}+\frac{1}{2} y^{3} e^{2 y^{4}}\left(x^{1} y^{5}+x^{2}\right)^{2} .
$$

Concluding the example we find the components of the function $\Psi(q, z)$ that defines the action of the local group $G_{e}$ on the homogeneous space $M=H \backslash G_{e}$, where $H=\exp (\mathfrak{h})$ with $\mathfrak{h}=\left\{e_{4}, e_{5}\right\}$. For this purpose, we choose $q^{1}=x^{1}, q^{2}=x^{2}, q^{3}=x^{3}, q^{4}=x^{6}$ as local coordinates on $M$. Then in view of $(2.16)$ we get

$$
\begin{aligned}
& \Psi^{1}(q, y)=q^{1} e^{-y^{4}}+y^{1}+y^{3} e^{y^{4}}\left(q^{2}+q^{1} y^{5}\right), \\
& \Psi^{2}(q, y)=e^{y^{4}}\left(q^{2}+q^{1} y^{5}\right)+y^{2}, \\
& \Psi^{3}(q, y)=\frac{e^{-2 y^{4}} q^{3}+y^{3}\left(1+q^{3} y^{5}\right)}{1+q^{3} y^{5}}, \\
& \Psi^{4}(q, y)=q^{4}+y^{6}+\frac{1}{2}\left(q^{1}\right)^{2} y^{5}+y^{2} y^{3} e^{y^{4}}\left(q^{1} y^{5}+q^{2}\right)+q^{1} y^{2} e^{-y^{4}}+\frac{1}{2} y^{3} e^{2 y^{4}}\left(q^{1} y^{5}+q^{2}\right)^{2} .
\end{aligned}
$$

\section{Transition from second canonical coordinates to first canonical coordinates}

The first canonical coordinates are universal in the sense that if one knows a composition function, then the transition to any type of canonical coordinates can be found and, consequently, the composition function can be represented in these coordinates. Consider the mentioned transition to the second canonical coordinates.

Let $g_{y}^{\mathrm{I}}$ be a group element in the first canonical coordinates $y^{i}$ and $g_{x}^{\mathrm{II}}$ is the same element in the second canonical coordinates $x^{i}$. The connection between the coordinate systems $y^{i}=Y^{i}(x)$ and $x^{i}=X^{i}(y), X=Y^{-1}$, follows from the equality

$$
\exp \left(\sum_{i=1}^{n} y^{i} e_{i}\right)=\prod_{i=1}^{n} \exp \left(x^{i} e_{i}\right) .
$$

Using (2.13), we multiply the exponentials and get

$$
\begin{aligned}
& Y^{i}(x)=\Phi^{i}\left(X_{1}, \Phi\left(X_{2}, \ldots\right) \ldots\right), \\
& X_{1}=\left(x^{1}, 0, \ldots, 0\right), \quad X_{2}=\left(0, x^{2}, 0, \ldots\right), \quad \ldots, \quad X_{n}=\left(0, \ldots, 0, x^{n}\right) .
\end{aligned}
$$


So, if we know the composition function $\Phi^{\mathrm{I}}$ in the first canonical coordinates, then the composition function $\Phi^{\mathrm{II}}$ in the second canonical coordinates can be easily obtained,

$$
\Phi^{\mathrm{II}}(x, \tilde{x})=X\left(\Phi^{\mathrm{I}}(Y(x), Y(\tilde{x}))\right) .
$$

The purpose of this section is to find the connection between the first and the second canonical coordinates in the case of unknown composition function.

Consider a local one-parametric subgroup

$$
\exp (t Y)=g_{y_{t}}^{\mathrm{I}}=g_{x_{t}}^{\mathrm{II}}, \quad Y=\sum_{i=1}^{n} y^{i} e_{i} .
$$

The equation defining this one-parametric curve in the first canonical coordinates is simple: $y_{t}^{i}=t y^{i}$. Let $x_{t}=X\left(y_{t}\right)=X(t y)=\alpha(t)$ be the equation of the same curve in the second canonical coordinates. As the zero coordinates correspond to the identity element of the group, we have

$$
\left.x_{t}\right|_{t=0}=\alpha(0)=0 .
$$

The variables $x$ and $y$ are coordinates of the same group element, which is equivalent to the condition

$$
\left.x_{t}\right|_{t=1}=\alpha(1)=x .
$$

It is well known that any one-parametric subgroup (as a curve in a group) is the integral trajectory of a left-invariant (resp. right-invariant) vector field. Consider the left- and rightinvariant vector field with a chosen direction $Y \in \mathfrak{g}$ at the identity element of the group, $\xi(x)=y^{i} \xi_{i}(x)$ and $\eta(x)=-y^{i} \eta_{i}(x)$. Since the trajectory of such a vector field is uniquely defined by the initial point and the associated direction at the identity element, we can find the equation describing the one-parametric subgroup as the solution of one of the systems of ordinary differential equations

$$
\begin{aligned}
& \frac{d x_{t}}{d t}=\xi\left(x_{t}\right), \\
& \frac{d x_{t}}{d t}=\eta\left(x_{t}\right),
\end{aligned}
$$

that additionally satisfies the initial condition (5.2). This solution parametrically depends on the variables $y^{i}, x_{t}=\alpha(t, y)=\alpha(1, t y)$. Using (5.3) we get the requested connection $x=X(y)=$ $\alpha(1, y)$ between the first and the second canonical coordinates.

Subtracting (5.5) from (5.4), we obtain the integrals of motion

$$
y^{i}\left(\xi_{i}^{k}\left(x_{t}\right)+\eta_{i}^{k}\left(x_{t}\right)\right)=0 .
$$

Using (2.8), this formula can be written as

$$
\operatorname{Ad}_{g_{x_{t}}^{\mathrm{II}}} Y=Y .
$$

The above integrals of motion simplify the integration of the system (5.4) or (5.5) but do not allow to get all the functions $\alpha^{i}(t, y)$. Show that this problem can be solved by quadratures and do not require any integration of the differential equations.

Proposition 2. The transformation $x=X(y)$ connecting the first and second canonical coordinates are found by quadratures. 
Proof. We replace the basis elements of the Lie algebra in (5.1) by their adjoint representations, $e_{i} \rightarrow$ ad $e_{i}$, which gives the matrix equality

$$
\operatorname{Ad}_{g_{y}^{\mathrm{I}}}=\operatorname{Ad}_{g_{x}^{\mathrm{II}}} .
$$

Recall that the exponentiation of a matrix is a linear algebra problem. Let $\mathfrak{z}$ be the kernel of the adjoint representation of the Lie algebra $\mathfrak{g}$. Denote by $\left(y^{\mu}\right),\left(x^{\mu}\right),\left(y^{a}\right)$ and $\left(x^{a}\right)$ the first and the second canonical coordinates of the $\operatorname{subgroup} \exp (\mathfrak{z})$ and the first and the second coordinates of the local quotient group $G_{e} / \exp (\mathfrak{z})$, respectively. For any element $Z \in \mathfrak{z}$, its adjoint representation ad $Z$ is the zero matrix. Hence the coordinates $y^{\mu}$ and $x^{\mu}$ are not involved in (5.6). In other words, the equation (5.6) connects the coordinates of the local quotient group $G_{e} / \exp (\mathfrak{z})$, which leads to $n$ - $\operatorname{dim} \mathfrak{z}$ components of the $n$-component function $X(y), x^{a}=X^{a}(y)$. Therefore, if the Lie algebra is centerless, then the equality (5.6) gives the complete solution. Otherwise we need to construct dimz more components $X^{\mu}(y)$.

It is easy to show that in any system of canonical coordinates, components of invariant vector fields and 1-forms do not depend on the coordinates $x^{\mu}$ of the center. Suppose that we have already computed the functions $X^{a}(y)$ using (5.6) and the components of left-invariant vector fields in the second canonical coordinates using, e.g., techniques developed in Section 2. Then the equations of the system (5.4) that are associated with the center can be easily integrated,

$$
x_{t}^{\mu}=X^{\mu}(t y)=\int_{0}^{t} \xi^{\mu}(X(t y)) d t=\int_{0}^{t} y^{i} \xi_{i}^{\mu}(X(t y)) d t
$$

since the expressions $\xi_{i}^{\mu}(X)$ do not involve $X^{\mu}$. Taking into account the condition (5.3), we get the rest of transformation components

$$
x^{\mu}=X^{\mu}(y)=\int_{0}^{1} y^{i} \xi_{i}^{\mu}(X(t y)) d t .
$$

The proposition is proven.

We illustrate Proposition 2 with the same six-dimensional Lie algebra $\mathfrak{g}$, which is defined by the commutation relations (3.5). As the center $\mathfrak{z}$ of $\mathfrak{g}$ is nontrivial, $\mathfrak{z}=\left\{e_{6}\right\}$, the matrix equality (5.6) connects the first and the second canonical coordinates on the quotient group $G_{e} / \exp (\mathfrak{z})$ as $x^{i}=X^{i}(y), i=1, \ldots, 5$. Specifically,

$$
\begin{aligned}
& x^{1}=y^{1} \sinh (J) / J+2\left(y^{2} y^{3}-y^{1} y^{4}\right) \sinh ^{2}(J / 2) / J^{2}, \\
& x^{2}=y^{2} \sinh (J) / J+2\left(y^{2} y^{3}+y^{1} y^{5}\right) \sinh ^{2}(J / 2) / J^{2}, \\
& x^{3}=y^{3}\left(\sinh (2 J) /(2 J)+y^{4} \sinh ^{2}(J) / J^{2}\right) /\left(\cosh (J)+y^{4} \sinh (J) / J\right)^{2}, \\
& x^{4}=\ln \left(\cosh (J)+y^{4} \sinh (J) / J\right), \\
& x^{5}=y^{5} \sinh (J) /\left(J \cosh (J)+y^{4} \sinh (J)\right), \quad J:=\sqrt{\left(y^{4}\right)^{2}+y^{3} y^{5}}
\end{aligned}
$$

The expressions for invariant fields (3.7) allow us to write down the integrand in (5.7) as

$$
y^{i} \xi_{i}^{6}(X(t y))=y^{2} X^{1}(t y)+y^{3} X^{2}(t y) / 2+y^{5}\left(X^{1}(t y)\right)^{2} / 2+y^{6},
$$

where

$$
\begin{aligned}
& X^{1}(t y)=y^{1} \sinh (t J) / J+2\left(y^{2} y^{3}-y^{1} y^{4}\right) \sinh ^{2}(t J / 2) / J^{2} \\
& X^{2}(t y)=y^{2} \sinh (t J) / J+2\left(y^{2} y^{3}+y^{1} y^{5}\right) \sinh ^{2}(t J / 2) / J^{2}
\end{aligned}
$$


The integral in (5.7) is easily computed in this case, which gives

$$
\begin{aligned}
x^{6}= & y^{6}+\frac{y^{1} y^{2} \cosh (J)-y^{1} y^{2}-\left(y^{2}\right)^{2} y^{3} / 2+\left(y^{1}\right)^{2} y^{5} / 2+y^{1} y^{2} y^{4}}{J^{2}}+ \\
& +\frac{\sinh (2 J)\left(\left(y^{2}\right)^{2} y^{3}+\left(y^{1}\right)^{2} y^{5}\right) / 4-\sinh (J)\left(y^{1} y^{2} y^{4}+\left(y^{1}\right)^{2} y^{5}\right)}{J^{3}}+ \\
& +\frac{\left(-2 y^{1} y^{2} y^{3} y^{5}-\left(y^{2}\right)^{2} y^{3} y^{4}+\left(y^{1}\right)^{2} y^{4} y^{5}\right)(\cosh (J)-\cosh (2 J) / 4-3 / 4)}{J^{4}} .
\end{aligned}
$$

We should note that the relation $x=X(y)$ is invertible and we can get the inverse mapping $y=Y(x)$ in elementary functions as well.

\section{Acknowledgements}

Authors greatly appreciate the cooperation of the editors and referees who put decent effort and amount of time to improve the content and style of the paper. We also want to especially thank the referees for the helpful discussions on the subject of the paper which moved our understanding of the problem much further. This work was supported by the Ministry of Education and Science of the Russian Federation (Project no. 3107).

\section{References}

[1] Alhassid Y., Engel J., Wu J., Algebraic approach to the scattering matrix, Phys. Rev. Lett. 53 (1984), 17-20.

[2] Baranovskii S.P., Shirokov I.V., Extensions of vector fields on Lie groups and homogeneous spaces, Theoret. and Math. Phys. 135 (2003), 510-519.

[3] Basarab-Horwath P., Lahno V., Zhdanov R., The structure of Lie algebras and the classification problem for partial differential equations, Acta Appl. Math. 69 (2001), 43-94, math-ph/0005013.

[4] Chevalley C., Theory of Lie groups, Vol. 1, Princeton University Press, Princeton, NJ, 1946.

[5] Cohn P.M., Lie groups, Cambridge Tracts in Mathematics and Mathematical Physics, Vol. 46, Cambridge University Press, New York, 1957.

[6] Draisma J., Transitive Lie algebras of vector fields: an overview, Qual. Theory Dyn. Syst. 11 (2012), 39-60, arXiv:1107.2836.

[7] Fushchich V.I., Barannik L.F., Barannik A.F., Subgroup analysis of Galilean and Poincaré groups and reduction of nonlinear equations, Naukova Dumka, Kiev, 1991.

[8] Fushchych W.I., Zhdanov R.Z., Symmetries of nonlinear Dirac equations, Mathematical Ukraina Publisher, Kyiv, 1997, math-ph/0609052.

[9] Goncharovskii M.M., Shirokov I.V., An integrable class of differential equations with nonlocal nonlinearity on Lie groups, Theoret. and Math. Phys. 161 (2009), 332-345.

[10] González-López A., Kamran N., Olver P.J., Lie algebras of vector fields in the real plane, Proc. London Math. Soc. 64 (1992), 339-368.

[11] Gorbatsevich V.V., Onishchik A.L., Lie groups of transformations, in Lie Groups and Lie Algebras, Current Problems in Mathematics. Fundamental Directions, Vol. 20, VINITI, Moscow, 1988, 103-240.

[12] Hausner M., Schwartz J.T., Lie groups; Lie algebras, Gordon and Breach Science Publishers, New York London - Paris, 1968.

[13] Heredero R.H., Olver P.J., Classification of invariant wave equations, J. Math. Phys. 37 (1996), 6414-6438.

[14] Kurnyavko O.L., Shirokov I.V., Construction of invariant wave equations of scalar particles on Riemannian manifolds with external gauge fields, Theoret. and Math. Phys. 156 (2008), 1169-1179.

[15] Lie S., Theorie der Transformationsgruppen, Vols. 1-3, Teubner, Leipzig, 1888, 1890, 1893.

[16] Lychagin V.V., Classification of the intransitive actions of Lie algebras, Soviet Math. (1989), no. 5, 25-38. 
[17] Miller Jr. W., Lie theory and special functions, Mathematics in Science and Engineering, Vol. 43, Academic Press, New York - London, 1968.

[18] Mosolova M.V., A new formula for $\ln \left(e^{A} e^{B}\right)$ in terms of the commutators of the elements $A$ and $B$, Math. Notes 23 (1978), 448-452.

[19] O'Cadiz Gustad C., Local structure of 2 dimensional solvable lie algebra actions on the plane, Lobachevskii J. Math. 33 (2012), 317-335.

[20] Petrov A.Z., New methods in the general theory of relativity, Nauka, Moscow, 1966.

[21] Pontryagin L.S., Continuous groups, Nauka, Moscow, 1973.

[22] Popovych R.O., Boyko V.M., Nesterenko M.O., Lutfullin M.W., Realizations of real low-dimensional Lie algebras, J. Phys. A: Math. Gen. 36 (2003), 7337-7360, math-ph/0301029.

[23] Shapovalov A.V., Shirokov I.V., Noncommutative integration of linear differential equations, Theoret. and Math. Phys. 104 (1995), 921-934.

[24] Shchepochkina I.M., How to realize a Lie algebra by vector fields, Theoret. and Math. Phys. 147 (2006), 450-469, math.RT/0509472.

[25] Shirokov I.V., Construction of Lie algebras of first-order differential operators, Russian Phys. J. 40 (1997), $525-530$.

[26] Terzis P.A., Faithful representations of Lie algebras and homogeneous spaces, arXiv:1304.7894. 